\title{
SEXUALIDADE DEPRESSIVA: REALIDADE PRESENTE E FUTURA
}

Carlos Pol Bravo ${ }^{1}$

\author{
DEPRESSED SEXUALITY: \\ PRESENT AND FUTURE REALITY
}

Resumo: Disfunções sexuais, foram levadas consideradas por muito tempo como aquelas "doenças secretas que se padeciam em segredo". Em prática médica temos pacientes com sintomatologias comuns, mas etiología diferente. Problemática sexual apresentando sintomatologia depressiva: Anhedonia, apatia, baixa auto-estima, sentimentos de perda, incapacidade, culpa, insegurança em sua conduta, celotipias, produzidas ante problemática sexual em fases: Desejo - Excitação - Orgasmo. Uma patologia depressiva como tal, mais disfunção sexual, são suficiente argumento Psicosociosexual para cristalizar dita sintomatologia. Não tentamos desconhecer a realidade de diminuição na libido em pacientes depressivos, mas si devemos valorizar porquê certas circunstâncias sexológicas, em sujeitos não diagnosticados como depressivos, apresentam sintomatologia denominada SEXUALIDADE DEPRESSIVA, similar em referências e queixas do paciente à depressão, mas diferente etiología: preocupação, ansiedade, decaimento frente ao problema: uma disfunção declarada e não valorizada em sua profundidade necessária para um tratamento em sexología médica. Aqui se precisa a história Psicosexual dentro a própria história clínica.

Palavras-chave: Disfunçōes sexuais; sintomatologia depressiva; históriapsicosexual.

Abstract: Sexual Dysfunctions has been taken till today, like those "secret diseases that were suffered in secret". In medical practice we have patients

\footnotetext{
Medico Psiquiatra - Master em Sexologia. Membro Ativo Associação Européia de Psiquiatria A.E.P. Presidente Fundador Sociedade Médica Espanhola de Sexología. Diretor Científico Central Médica de Sexología - Bogotá. Membro Fundador Academia de Ciências Medicas do Clube de Medico - Bogotá. e-mail: centralmedicasexologia@yahoo.es
} 
with common sytonms, but different etiology. Sexual Problematic presenting depressive sinthomatology: Anhedonia, apathy, low self-esteem, feelings of lost, incapacity, blame, insecurity in its conduct, celotipic problems, produced in front of sexual problems in the three phases: Desire - Excitation - Orgasm. A depressive pathology like as so, plus a sexual dysfunction, are sufficient Psicosociosexual argument to crystallize this sinthomatology. We did not try to unknown the reality of diminution of the libido in depressive patients, but we must value why certain sexológical circumstances, in subjects non diagnosed like depressive, presents the denominated DEPRESSIVE SEXUALITY similar in references and complaints of the patient to the depression, but different etiology: Preoccupation, anxiety, decay as opposed to the problem: A dysfunction declared and not valued in its necessary depth for a treatment in medical sexology. Here Psicosexual history needs to bee inside on the own clinical history.

Keywords: Sexual dysfunctions; depressive sinthomatology; psicosexual history.

Faz mais de um século León Tolstoy dizia como "O ser humano estava preparado para sobreviver às guerras, as epidemias, os horrores da doença $\mathrm{e}$ as torturas do Espírito. Mas que a tragédia mais atormentadora de todos os tempos, foram e são as tragédias de alcova." (ref.???)

As disfunções sexuais foram levadas também até a data como "aquelas doenças secretas, que se padeciam em segredo.

"E nos baseamos nesse conceito entre apaixonado e melancólico da literatura Russa, para entrar no marco Clínico da sexualidade e a depressão, podendo assim deixar a inquietude que centra nosso trabalho com o seguinte dilema:

Existe a diminuição da libido na depressão tão só como um sintoma da mesma, ou existe também a diminuição da atividade sexual que ao passo do tempo se cimienta, e impede uma comunicação quando menos llevadera nas relações sexuais e sociais do paciente, talvez pela falta de informação terapêutica adequada com respeito a seu problema sexual, ou de uma Psicoeducación oportuna em referência ao caso?

Ou por outra parte nos encontramos ante uma Disfunção Sexual (D. S.) propriamente dita e pontualmente angustiante para o varão como é a 
disfunção eréctil, cuja instauração como explicaremos mas adiante, levalhe a expressar uma sintomatologia depressiva?

Igualmente é valido propor o caso de uma mulher com falta de lubrificação durante a fase de Excitação, levando-a a uma diminuição do Desejo sexual podendo apresentar anhedonia, introversión, perda de autoestima, etc.

H. Kaplan em seu livro sobre a Avaliação de Transtorno Sexuais (1988), suscita as seguintes inquietudes:

“Que foi primeiro, a depressão ou o sintoma sexual?

- Foi a depressão subjacente a que motivo que o paciente perdesse a libido, ou foi a perturbação sexual o que a deprimiu e sumiu posteriormente num estado de pesar?

- Tratando-se de uma mulher: deve considerar-se a dificuldade para realizar o coito como um sinal da depressão, ou foi a disfunção sexual causada por uma secura vaginal e a subseqüente frustração em suas relações sexuais o que a precipito à depressão?

Pelo mesmo eixo causa - efeito, nós dizemos:

A um varão com disfunção eréctil; sua ansiedade anticipatoria de fracasso não pode levar-lhe a dificuldades na fase de Desejo e tudo junto a condutas evitacionistas nas relações sexuais, precipitando-o também a uma sintomatologia depressiva, por sentimentos de incapacidade ou culpa?

Evidentemente não tentamos desconhecer a realidade sintomática da depressão sobre a sexualidade em pacientes diagnosticados como tais. Mas si enfatizar o porquê, em certas ocasiōes a conduta sexual em sujeitos não diagnosticados como depressivos, e por fatores determinados ou bem desconhecidos, transforma-se no que bem poderíamos chamar "SEXUALIDADE DEPRESSIVA."

A grande problemática Clínica nestes casos é averiguar detidamente, o possível transtorno depressivo, ou a alteração na conduta sexual, que pode ser ocasionado por uma disfunção - mantida e não descoberta - afundando na esfera sexual do paciente e seu casal se a tem, para estabelecer um diagnostico diferencial correto:

Estamos ante uma depressão cuja sintomatologia é concomitante a uma disfunção sexual quanto à conduta sexual do paciente se refere, ou ante esta propriamente dita em qualquer de suas fases: Desejo sexual inibido, dificultade 
de lubrificação vaginal, falta de ereção, eyaculación precoce, anorgásmia ou eyaculación retardada.

Todas podem levar a uma relação sexual conflitiva, sem gratificação, e cuja reiterada frustração ou fracasso, ao ser referidas pelo paciente sobre seu estado de animo: Queixas somáticas, sentimentos de culpa e insegurança junto com apatia, anhedonia, abulia e falta de interesse pelo sexo, sugeririam certas suspeitas de sintomatologia depressiva.

Estas situações são valorizadas, mas pouco aprofundadas numa alta percentagem de Histórias Clínicas quanto ao aspecto da esfera sexual se refere.

Ante a carência de perspectivas satisfatórias não existe gratificação sexual, e a dinâmica que pode manter um desejo ativo se deteriora começando a criar resistências com respeito ao ato sexual, o que afeta tanto ao paciente como a seu casal chegando ao que chamaríamos de novo como "sexualidade depressiva" e diminuição na qualidade de vida.

Em certas ocasióes o quadro se recrudece pela falta de apoio que o casal queira ou possa brindar-lhe, segundo seus conhecimentos ou crenças sobre o sexo.Em outros casos, a dificuldade de comunicação e asertividad social impedem estabelecer boas relações sociais do afetado, criando um feedback em sua problemática Psicosociosexual.

Por outra parte, ainda que falemos sempre de "o casal", não é necessário que um paciente quem apresenta estas características a tenha. Costumam ocorrer estas situações indistintamente em pessoas com ou se parelha, e apresentar evidentemente as mesmas queixas e / ou sintomatologia. Os fatores desencadeantes ou de manutenção podem ser o único ponto de partida para abordar mas exatamente o problema que apresenta o paciente.

Com a intenção de reafirmar-nos em nossa teoria, existe o conceito de autores como R. Taylor no Simposium de Terapia sobre antidepressivos e seus efeitos secundários nas D. S. (Virginia, 1992.) quem explica como uma vez o clínico ao reconhecer um problema sexual num paciente tratado por um transtorno afetivo, encontra-se com um dilema típico envelope diagnostico:

- Este problema é secundário ao transtorno afetivo?

- É secundário à farmacoterapia para dito transtorno?

- Ou é devido a problemas de casal (o qual a sua vez pode ser secundário a um transtorno depressivo ou relacionado a um ou vários fatores produzidos por uma alteração física?). 
De novo nos questionamos o tema dizendo:

Pode o problema sexual ser primário à possível depressão, desencadeá-la ou potenciá-la?

De ser positivo o proposto por Taylor, é necessário indubitavelmente dar maior importância ao aspecto sexual para do que o Psiquiatra possa abordar em profundidade o caso, e efetuar uma valoração mas profunda através de uma história psicosexual bem estruturada sobre os conhecimentos de sexualidade em general do paciente, e se padece um D. S. desta em particular.

Em tal caso, conscientiza-lo de sua problemática sexual devido ao D. S. e efetuar o plano de trabalho adequado, pautando o devido tratamento farmacológico se procede, junto à devida terapia sexual de apoio, o que pode mudar o horizonte psicossocial do paciente

Assim se facilita o diagnostico diferencial bem no campo sexológico, ou o psiquiátrico, com o correspondente tratamento antidepressivo, guardando sempre por norma que de ser um quadro de etiología depressiva, tem de tratar-se primeiro a depressão e depois o problema sexual.

\section{CONDUTA SEXUAL NA DEPRESSÃO}

Uma vez explicados os conceitos da " sexualidade depressiva, “ sua ambígua sintomatologia e busca de diagnostico, quiséssemos fazer uns breves comentários sobre a sexualidade dentro a depressão.

O enfermo depressivo sofre umas mudanças por todos conhecidos na esfera sexual como em outras tantas. Mas esta quiçá é uma pedra angular pela importância que tem, já que afeta uma das principais formas de comunicação humana.

Segundo o conceito de Beck (1967) sobre a influência dos transtornos psiquiátricos e as disfunções sexuais nos pacientes que padecem depressão, em sua maioria estes experimentam uma perdida de seu interesse sexual.

Weissman e Paykel (1974) encontraram que com respeito à sexualidade, a diferença mas pronunciada entre as mulheres deprimidas e as não deprimidas, era o grau da perdida do interesse ou desejo sexual nas primeiras. Umas poucas referiram disfunção orgásmica ou disparéunia. Ao recuperar-se da depressão, a mudança importante no ajuste sexual foi um aumento de interesse pelo mesmo. 
O trazer estas definições de autores bem conhecidos, é para separar claramente a depressão e sua conseqüente diminuição da libido, do conceito apresentado: Sexualidade depressiva. Com sintomatologia similar, mas etiología diferente.

\section{FARMACOS ANTIDEPRESSIVOS.}

Analisando o problema sexualidade - depressão dentro um marco farmacológico, é indubitável a influência dos antidepressivos na conduta sexual, produzindo uma série de efeitos secundários bem conhecidos.

Podemos começar citando os efeitos de tipo periférico dos antidepressivos heterocíclicos segundo o Prof. E. González Monclús em seu livro Psicofarmacologia aplicada (1993)

"As disfunções sexuais se referem à dificuldade na ereção, ao retardo ou a inibição da eyaculación no varão. À diminuição da libido, e atraso no orgasmo ou anorgásmia feminina."

Para H. Kaplan (Disfunções sexuais 1988.) "A triste realidade é que os Psicotropos podem menoscabar o ciclo da resposta sexual nas fases de Desejo - Excitação e Orgasmo (D.E.O.) em função das doses e com referência a determinados pacientes."

Com freqüência os problemas sexuais são passados por alto, ou interpretados de forma equivoca.

Seguindo com os conceitos de Kaplan, o diagnostico diferencial e meticuloso dos estados de ansiedade e depressão em pacientes que sofrem D.S. é: “Um fator de vital importância. Daí a necessidade de um correto diagnostico, medicação e psicoterapia”

Esta pauta, reforça a idéia antes mencionada por nós, sobre a transcendência que pode ter uma maior abordagem na anamnese psicosexual do paciente.

Em alguns casos, certos pacientes advertem uma melhoria do humor depressivo, produzindo-se um aumento do desejo sexual e a respectiva gratificação, o qual pode ser potenciado pelo casal.

Em outros, podem produzir-se uma série de situações anómalas:

1. Rentismo, celotipias e insegurança no casal.

2. Uma disfunção sexual adquirida por concomitância com o casal depressivo, pode dificultar o novo horizonte psicosexual, que pode dissipar-se com 
um oportuno tratamento antidepressivo e sobre a disfunção eréctil, de ser esta o motivo central.

Assim mesmo J. Gitlin (UCLA. School of Medicine, Los Angeles: California.) em seu Articulo sobre os possíveis efeitos secundários na conduta sexual de pacientes tratados com antidepressivos, explica como as diferenças em ambos sexos não foram o suficientemente estudadas, sendo provável que tanto varôes como mulheres estejam afetados igualmente pelos efeitos secundários no aspecto sexual.

Dos três estudos que classificam os níveis de D. S. por antidepressivos segundo o sexo, com um deles se encontrou um alto nível de disfunçōes nas mulheres.

Outro estudo era mas igualado, e o terceiro deu maior nível de afectação nos varōes.

Não obstante as mulheres pareciam mais espontâneas em comunicar estes efeitos secundários da esfera sexual.

Adicionalmente, quiçá devido à maior facilidade da observação na função eréctil do varão, bastante estudos se enfocaram exclusivamente nas funçōes sexuais masculinas, especialmente a disfunção eréctil.

Nas três classes de Psicotropos mas comummente prescritos: Antidepressivos, Benzodiacepinas e Neurolépticos, cada um deles costuma ir sócio com um efeito secundário em cada área da função sexual.

Vemos como alguns Fármacos que podem ter um efeito direto sobre o cérebro, costumam interferir no interesse (Desejo) sexual ainda que dito efeito seja secundário às mudanças do estado de animo. (Ej: Metildopa.)

Quiçá a causa mais comum sobre os efeitos secundários na sexualidade, é a interferência nas vias nervosas autonômicas, relacionadas no processo de ereção e lubricação ou eyaculación e orgasmo (Fases E.O.) como são: Antidepressivos de primeira e segunda geração e os tranquilizantes tanto maiores como menores.

É bastante difícil valorizar os efeitos dos antidepressivos na sexualidade, devido às sérias alterações que pode provocar a depressão sobre a conduta sexual em muitos varōes e mulheres.

Para concluir, cremos interessante recordar os Estudos de Beaumont (1977) assinalando que a diminuição da libido foi o efeito mais comum da depressão tanto em varôes como em mulheres. 
Iniciado o tratamento com/ A D. (Clorimipramina) efetuaram-se mudanças na conduta sexual dos pacientes.

Uma quinta parte dos varōes referiram dificuldades na ereção e algumas mulheres começaram a sofrer dificuldades para atingir o orgasmo.

No entanto outras melhoraram sua resposta sexual com a ingesta da A/D. provavelmente pelo efeito benéfico destes sobre seu estado de animo.

Segundo K. Hawton, (1988) em relação aos estados de ansiedade, deixa a inquietude de que não se conhece tão a fundo como séria desejável, a forma como afetam à sexualidade em seu Ciclo de resposta normal.

Depois de uma profunda revisão chegou à surpreendente impressão de que os efeitos não são tão profundos, sobretudo comparados com a depressão.

Isto pode dever-se a que é a ansiedade vinculada à sexualidade, e não a ansiedade geral, a causadora de muitos problemas sexuais.

\section{A MODO DE CONCLUSÕES}

Baseando-nos nesse duplo conceito, animamo-nos a incidir uma vez mas em como a depressão e a própria ansiedade vinculada à sexualidade, ou uma forma de conduta sexual deficitária em conhecimentos, educação e imaginação, pode levar a uma sexualidade mermada e com sérias tendências a uma diminuição do interesse ou desejo sexual, como chave primeiramente a outras disfunçōes 'pela própria apatia que implicam tais atitudes e as repercussōes psicossociais produzidas no casal.

Por conseguinte, chegam a confundir-se nesse marco claroscuro de uma depressão solapada, com todas suas conseqüências.

Por isso cremos positivo basear o sucesso diagnostico numa abordagem incisiva e profunda sobre a esfera sexual desses pacientes e seus casais:

- Transculturalidad, tipo de educação sexual, possíveis traumas. hábitos, etc. - Ter presente a dimensão básica da classificação descrita por Masters \& Johnson sobre o início do problema e as circunstâncias em que ocorre, sendo Primárias ou secundárias para o início e situacionales ou totais para as Circunstâncias.

- Posteriormente Kaplan desenvolve este conceito ao plasmar seu modelo trifásico Desejo - Excitação - Orgasmo, e como o desejo tende a bloquearse ante situaçóes especificas, ou com um colega em concreto. 
A transcendência desta classificação fica plasmada como base de estudo, ao ser introduzida no D. S. M. IV-.R. Para especificar os critérios diagnósticos sobre as D. S. - É também lógico compartilhar as acertadas atitudes gerais de variar as doses ou mudar o fármaco segundo as reações da conduta sexual naqueles pacientes afetados pelos efeitos secundários.

- Também é muito valido, incluir definitivamente em nossa baterista terapêutica o binômio $\mathrm{A} \mathrm{D} \mathrm{/} \mathrm{-} \mathrm{D.} \mathrm{E.} \mathrm{(fármaco} \mathrm{antidepressivo} \mathrm{-} \mathrm{fármaco} \mathrm{para} \mathrm{a}$ disfunção eréctil) com os novos medicamentos de administração oral a doses terapêuticas, e tendo o paciente a oportunidade de poder tomá-lo num tempo discreto antes de ter uma relação sexual, e cujos estudos e experiência Clínica permitem mudar em plenitude o conceito de "sexualidade depressiva” ou mínimo encurtar a problemática sexual para determinados casos em que exista um componente depressivo onde a disfunção eréctil afete especialmente a qualidade de vida do paciente masculino.

- Desta forma fechamos o ciclo da resposta sexual com o apoio terapêutico em suas múltiplas fases, sem esquecer nunca a importância que têm todas em seu complexo equilíbrio: Desejo - Excitação - Orgasmo. Onde o Modelo Biopsicosociosexual reestrutura o desenvolvimento de uma atividade sexual gratificante ou quando menos practicable no indivíduo afetado, e conseqüentemente em sua relação, melhorando assim a qualidade de vida sexual em ambos membros do casal.

\section{Referências bibliográficas}

BORRULL, J. Trastornos afectivos en la menopausia. P.T.D. Barcelona: Editorial Espax., 1991.

BRAVO, C.P. Interconsulta Psiquiátrica. In: ROJO, E.; CIRERA, E. Sistema Sexual y Reproductor

CIE 10. Apartado sobre Disfunciones sexuales Trastornos mentales y del Comportamiento. Forma S. A. Madrid, 1992..

D.S.M IV - R. Apartado sobre Trastornos sexuales y de la identidad sexual. Barcelona: editora Masson, 1995.

FREDMAN; SADOCK; KAPLAN. Sexualidad Humana normal y anormal. Tratado de Psiquiatria. Barcelona: Editorial Suramericana, 1982.

HAWTON, K. Terapia Sexual. causa de los problemas sexuales. Barcelona: Ediciones Doyma, 1988. 
KALINA, Z.; BEZCHLIBNYK, B. Clinical Handbook of psychotropic drugs. Fourth revised edition.. Toronto: Hogrefe \& Huber Publishers. Sattle. Göttingen. Bern, 1994.

KAPLAN, H.S. Disfunciones Sexuales. Trastornos sexuales y medicación. Barcelona: Ediciones Grijalbo S.A., 1988.

KAPLAN, H. S. Evaluación de los trastornos Sexuales. Evaluación médica de los trastornos del Deseo Sexual en el varón y la mujer. Barcelona: Ediciones Grijalbo S.A., 1985.

MASTERS \& JHONSON. El Vinculo del Placer. Barcelona:_Ediciones Grijalbo, 1985.

MICHAEL, J.; GITLIN, M.D. Psycotropic Medications and Their effects on Sexual Function: Diagnosis Biology and Treatment Aproaches. J. Clinical Psychiatry 55:9, September. 1994. UCLA School of Medicine. Psychiatryc Dept. University of California.

MONCLÚS, E.G. Psicofarmacologia Aplicada. - efectos secundarios de los Psicofarmácos. Barcelona: Organon Española, 1993.

SADOCK; KAPLAN. Sexualidad Humana. Tratado de Psiquiatria. $2^{a}$ edição. Barcelona: Editorial Suramericana, 1995.

SEGRAVES, R.T.M.D. Overview of Sexual Disfunction Complicating the Treatment of Depression. The Journal of Clinical Psychiatry, volume $10^{\text {th }}$. Sept., 1992.

VALLEJO, J.; BULBENA, A. Introducción a la Psicobiología y la Psiquiatría. 2a Edición. Barcelona: Salvat Editores, 1988. 